Article

\title{
How Can Synchrotron Radiation Techniques Be Applied for Detecting Microstructures in Amorphous Alloys?
}

\author{
Gu-Qing Guo, Shi-Yang Wu, Sheng Luo and Liang Yang *
}

College of Materials Science and Technology, Nanjing University of Aeronautics and Astronautics, Nanjing 210016, China; E-Mails: guoguqing@nuaa.edu.cn (G.-Q.G.); shiyangwu0914@gmail.com (S.-Y.W.); nuaaluosheng@163.com (S.L.)

* Author to whom correspondence should be addressed; E-Mail: yangliang@nuaa.edu.cn; Tel.: +86-25-52112903; Fax: +86-25-52112626.

Academic Editor: Klaus-Dieter Liss

Received: 23 September 2015 / Accepted: 2 November 2015 / Published: 4 November 2015

\begin{abstract}
In this work, how synchrotron radiation techniques can be applied for detecting the microstructure in metallic glass (MG) is studied. The unit cells are the basic structural units in crystals, though it has been suggested that the co-existence of various clusters may be the universal structural feature in MG. Therefore, it is a challenge to detect microstructures of MG even at the short-range scale by directly using synchrotron radiation techniques, such as X-ray diffraction and X-ray absorption methods. Here, a feasible scheme is developed where some state-of-the-art synchrotron radiation-based experiments can be combined with simulations to investigate the microstructure in MG. By studying a typical MG composition $\left(\mathrm{Zr}_{70} \mathrm{Pd}_{30}\right)$, it is found that various clusters do co-exist in its microstructure, and icosahedral-like clusters are the popular structural units. This is the structural origin where there is precipitation of an icosahedral quasicrystalline phase prior to phase transformation from glass to crystal when heating $\mathrm{Zr}_{70} \mathrm{Pd}_{30} \mathrm{MG}$.
\end{abstract}

Keywords: metallic glasses; extended X-ray absorption fine structure; X-ray diffraction; reverse Monte Carlo simulation; microstructure 


\section{Introduction}

The atomic structure of metallic glass (MG) is a long-standing issue and has been attracting great interest since the 1960s [1-7] because of its unique properties and forming ability which is strongly related to its atomic structure. The microstructure of MG is rather complex, and the three-dimensional structural picture in this class of alloys is far from being established. Fortunately, thus far, several structural models have been proposed theoretically, enhancing the understanding of the glass-forming mechanisms in binary MG by building and stacking clusters in space to reveal their short-range and medium-range orderings [5,8-10]. However, there is a challenge in probing the atomic-scale structure by using conventional experimental techniques. As an advanced experimental platform, a synchrotron radiation facility can provide a series of state-of-the-art techniques for detecting the microstructure of various materials, especial for some amorphous materials [11]. In the previous work, synchrotron radiation methods such as X-ray diffraction (XRD) and extended X-ray absorption fine structure (EXAFS) and neutron diffraction have been applied for studying MG [12-14]. Nevertheless, because XRD and EXAFS can only provide the average atomic distributions and the average surroundings of each kind of atom (element-specific), respectively, whether performing these synchrotron radiation experiments can directly reveal the complex microstructure of MG is a controversial issue $[15,16]$.

In this work, a feasible scheme for addressing this issue is developed by performing a series of state-of-the-art synchrotron radiation-based experiments combined with simulations to investigate the microstructures of amorphous alloys $[17,18]$. $\mathrm{Zr}_{70} \mathrm{Pd}_{30}$ binary alloy is selected as the research prototype due to the following reasons: (1) compared with multicomponent alloys, the $\mathrm{Zr}_{70} \mathrm{Pd}_{30}$ alloy has a relatively simple composition, enhancing the reliability of structural results; (2) an icosahedral quasicrystalline primary phase (I-phase) was detected when heating the $\mathrm{Zr}_{70} \mathrm{Pd}_{30} \mathrm{MG}$ [19], and it was suggested that icosahedral clusters are the basic building blocks in the microstructure of this glassy alloy [20]. Therefore, as a contrast, the icosahedral cluster can be used as the initial structural model to directly fit EXAFS signals.

\section{Experimental Section}

The $\mathrm{Zr}_{70} \mathrm{Pd}_{30}$ binary ingot was prepared by arc-melting high-purity metals $(99.9 \% \mathrm{Zr}$ and 99.9\% Pd) [21,22]. Amorphous ribbons with a cross-section of $0.04 \times 2 \mathrm{~mm}^{2}$ were produced from the ingot via single-roller melt spinning at a wheel surface velocity of $40 \mathrm{~m} / \mathrm{s}$ in purified Ar atmosphere. Firstly, X-ray diffraction (Cu $\mathrm{K}_{\alpha}$, radiation) and high-resolution electron microscopy measurements were performed to confirm the amorphous state of the as-prepared sample. Subsequently, room temperature X-ray diffraction (XRD) measurement was performed using a high-energy synchrotron radiation monochromatic beam (about $100 \mathrm{KeV}$ ) on beam line BW5 in Hasylab, Germany [23]. Two-dimension diffraction data was collected by a Mar345 image plate, and then was integrated to Q-space ( $Q$ is the wave vector transfer) after subtracting the corresponding background by using program Fit2D [24]. The output data was normalized by software PDFgetX to obtain structure factor S(Q) according to the Faber-Ziman equation [25]. Furthermore, extended X-ray absorption fine structure (EXAFS) measurements for $\mathrm{Zr}$ and Pd K-edge were carried out using transmission mode at beam lines BL14W1, in the Shanghai Synchrotron Radiation Facility of China and U7C, in the 
National Synchrotron Radiation Laboratory (NSRL) of China. These EXAFS raw data were normalized via a standard data-reduced procedure, employing the Visual Processing in EXAFS Researches (VIPER) [26].

In this work, two methods were applied for detecting the microstructure of $\mathrm{Zr}_{70} \mathrm{Pd}_{30} \mathrm{MG}$, based on the normalized EXAFS and XRD data. The first method is that we directly fit the $\mathrm{Zr}$ and the Pd K-edge EXAFS signals with the Zr- and the Pd-centered icosahedral cluster models simultaneously, using the software VIPER. According to our previous work [14], these two icosahedral models could be obtained by extracting some typical clusters from $\mathrm{ZrPd}$ binary crystalline phases, such as the $\mathrm{Zr}_{2} \mathrm{Pd}_{1}$ tetragonal phase. The second method is that rather than fitting the EXAFS signals directly, we simulated all the EXAFS and XRD data simultaneously under the framework of reverse Monte-Carlo (RMC) [27], because the RMC-simulation technique is an efficient iterative method for building a structural model in disordered systems with detailed structural information that agree quantitatively with experimental data (such as synchrotron radiation-based XRD and EXAFS, and neutron-diffraction data) [28].

\section{Results and Discussion}

When directly fitting the EXAFS signals with the Zr- and Pd-centered icosahedral cluster models, fixing and unfixing the coordination numbers (CNs) of the cluster models were both tried. Fixing the CNs of the cluster model to fit the EXAFS signal is usually adopted when the short-range ordering (local structure) of the measured sample resembles the cluster model. Because it was suggested that the icosahedral clusters are the building blocks in ZrPd binary MG, the icosahedral quasicrystalline primary phase could appear during annealing [19]. Thus, it seems reasonable to fit the EXAFS signals by fixing the CNs of the icosahedral cluster models. Figure 1 shows the $\mathrm{Zr}$ and the Pd K-edge EXAFS signals $\left(\kappa^{3} \cdot \chi(\kappa)\right)$, as well as their fitted data.
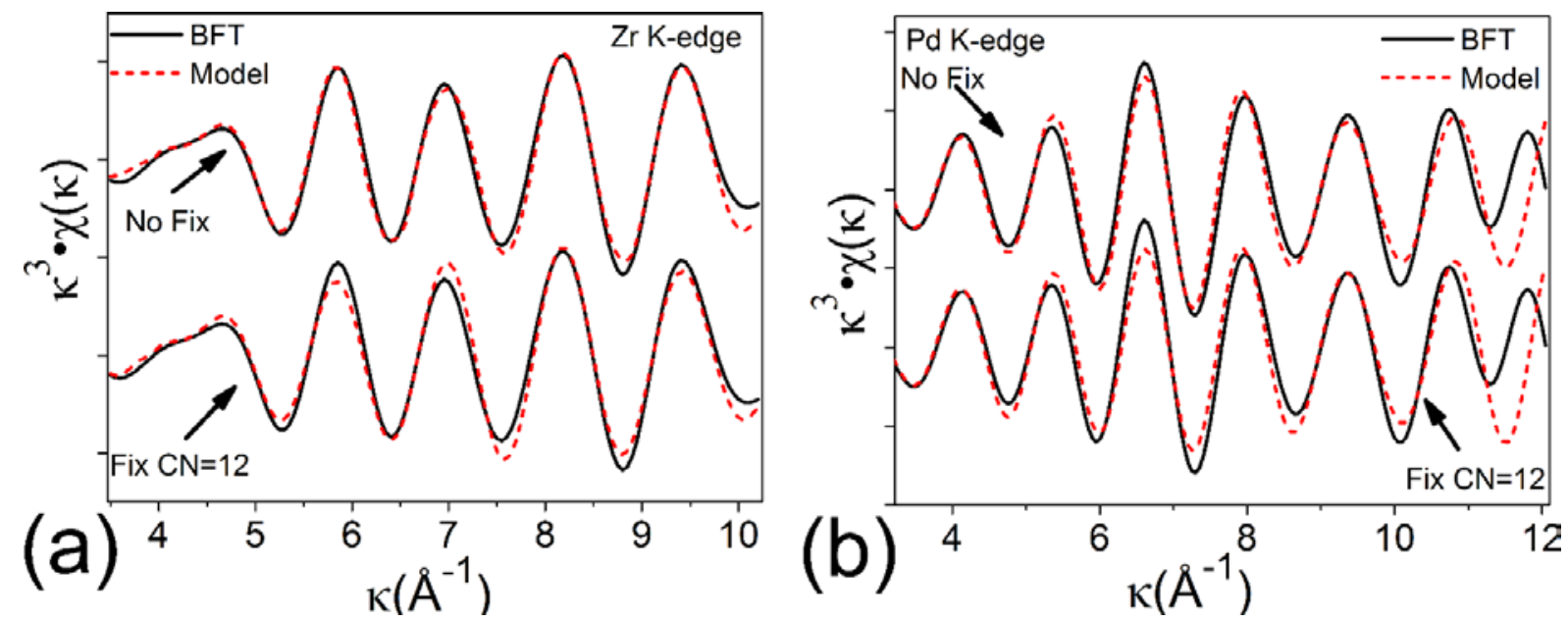

Figure 1. The fitted EXAFS signals of (a) $\mathrm{Zr}$ K-edge; and (b) Pd K-edge. The experimental and fitted data are plotted with solid and dashed lines, respectively. Here $\kappa$ is the wave vector, and the original EXAFS signal $(\chi(\kappa))$ is weighted by $\kappa^{3}$. The icosahedral cluster model is the initial fitting model. Here, fixing and unfixing the $\mathrm{CN}$ of icosahedral cluster models were both tried. 
These fitted Zr and Pd K-edge EXAFS curves are consistent with their experimental counterparts. All the fitted data are listed in Table 1, including the CNs around Zr or Pd centers, atomic distances $(R)$, energy shifts, and the relative displacement of atoms. These fitted data are some physical parameters that not only can reflect the short-range orderings in the microstructure of MG, but also can be applied for evaluating the success of the fitting itself. As shown in Table 1, when the total CNs around $\mathrm{Zr}$ or Pd centers are fixed, the energy shifts have abnormal values larger than $20 \mathrm{eV}$, which are seldom observed if the structural model can fit the experimental data properly. On the other hand, when the total CNs around $\mathrm{Zr}$ or Pd centers are not fixed, there are abnormal CN values far from that of the icosahedral cluster $(\mathrm{CN}=12)$. For instance, the $\mathrm{CN}$ of $\mathrm{Zr}$ centers $(6.0+2.9=8.9)$ is much smaller than 12 , while that of Pd centers $(2.8+17.1=19.9)$ is much larger than 12 . The abnormal values of these physical parameters indicate that it is not proper to fit the EXAFS signals of MG directly by using the icosahedral cluster model, whether the CN of this model is fixed or not.

Table 1. Atomic structural information obtained from the EXAFS fitting, including coordination numbers (CNs) around $\mathrm{Zr}$ or Pd centers, atomic distances $(R)$, energy shifts, and the relative displacement of atoms.

\begin{tabular}{|c|c|c|c|c|c|c|c|c|c|}
\hline \multirow{2}{*}{ Absorption Edge } & \multirow{2}{*}{$\begin{array}{c}\text { Fitting } \\
\text { Condition }\end{array}$} & \multicolumn{2}{|c|}{$R(\AA) \pm 0.02$} & \multicolumn{2}{|c|}{$\mathrm{CN} \pm 0.1$} & \multicolumn{2}{|c|}{$\sigma^{2}(\AA) \pm 0.001$} & \multicolumn{2}{|c|}{$E_{0}$ Shift $(\mathrm{eV}) \pm 0.01$} \\
\hline & & $\mathrm{ZrZr}$ & ZrPd & ZrZr & ZrPd & $\mathrm{ZrZr}$ & ZrPd & ZrZr & ZrPd \\
\hline \multirow{2}{*}{ Zr K-edge } & CN unfixed & 3.10 & 3.00 & 6.0 & 2.9 & 0.038 & 0.014 & 1.20 & -11.80 \\
\hline & CN fixed & 3.10 & 3.01 & 8.8 & 3.2 & 0.045 & 0.015 & 24.01 & -23.34 \\
\hline- & - & PdPd & $\mathrm{PdZr}$ & PdPd & $\mathrm{PdZr}$ & PdPd & $\mathrm{PdZr}$ & PdPd & $\mathrm{PdZr}$ \\
\hline \multirow{2}{*}{ Pd K-edge } & CN unfixed & 2.74 & 2.95 & 2.8 & 17.1 & 0.013 & 0.068 & -9.72 & -4.62 \\
\hline & CN fixed & 2.71 & 2.90 & 2.7 & 9.3 & 0.015 & 0.078 & -21.72 & -22.58 \\
\hline
\end{tabular}

Figure 2a shows the original two-dimensional X-ray diffraction pattern of $\mathrm{Zr}_{70} \mathrm{Pd}_{30}$ and Figure $2 \mathrm{~b}$ is the RMC-simulated structural model. Figure 2c-f show the simulated $S(Q)$ curve, the $G(r)$ curve, the $\mathrm{Zr}$ and Pd K-edge EXAFS spectra, as well as their corresponding experimental data. As shown in Figure 2a,c, i.e., the two-dimensional diffraction pattern and the one-dimensional diffraction data, except for a bright hole (sharp peak) located at about 2.5-3.5 $\AA^{-1}$ and some other halos (broad peaks) ranging from 4-6 $\AA^{-1}$ and 6-8 $\AA^{-1}$, there is no circle (sharp Bragg peak). Therefore, the full amorphous structure in this sample is confirmed. The good matching between all the experiment-simulation pairs confirms success of the RMC simulation. The simulated structural model shown in Figure $2 b$ contains a mass of position-determined atoms, so that atomic- and cluster-level structural information can be deduced accordingly. On the atomic scale, the nearest atomic-pair distances are listed in Table 2. Such data are compared with the sum of their Goldschmidt atomic radii (SGAR) and those extracted from the corresponding crystalline compounds, such as the $\mathrm{Zr}_{2} \mathrm{Pd}_{1}$ tetragonal phase obtained by annealing treatment on the $\mathrm{Zr}_{70} \mathrm{Pd}_{30} \mathrm{MG}$ [19]. Atomic-pair distances in the $\mathrm{Zr}_{2} \mathrm{Pd}_{1}$ I-phase are not provided here due to the lack of such values. It is found that the $\mathrm{Zr}$-Pd bond apparently differs from both values of the SGAR and the tetragonal phase so that its length is shortened by $0.1-0.2 \AA$. This strongly suggests that a strong interaction between $\mathrm{Zr}$ and $\mathrm{Pd}$ atoms occurs in the $\mathrm{Zr}_{70} \mathrm{Pd} 30$ binary glassy alloy, which is consistent with the previous work [29], in particular the findings of the d-band bonding changes with the transition metals [30,31]. 

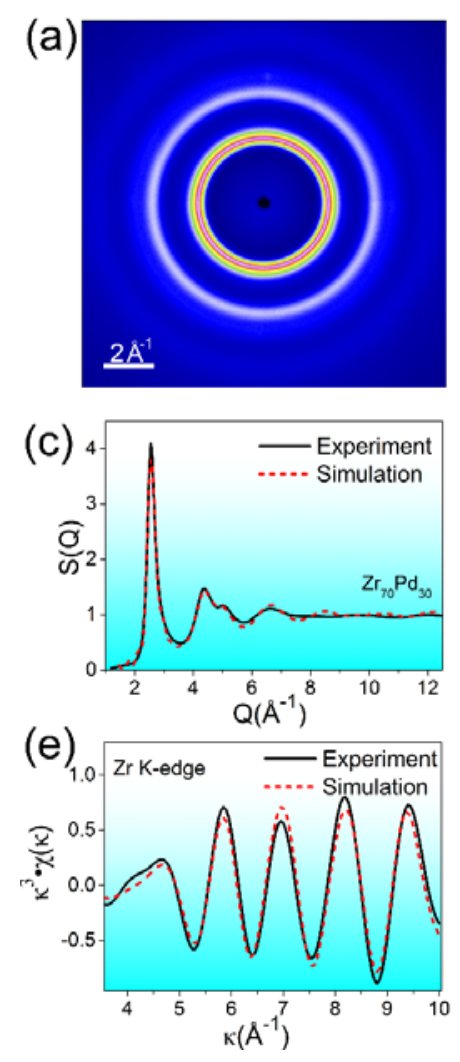
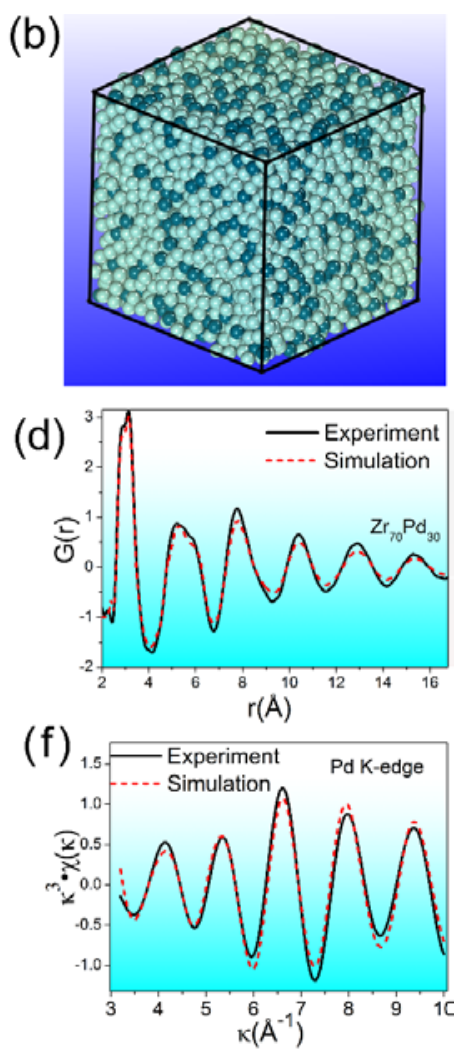

Figure 2. (a) The original two-dimensional X-ray diffraction pattern of $\mathrm{Zr}_{70} \mathrm{Pd}_{30}$ and (b) the RMC-simulated structural model. (c), (d), (e), and (f) are the RMC-simulated $S(Q)$, $G(r), \mathrm{Zr}$ K-edge, and Pd K-edge EXAFS signals, as well as their corresponding experimental data. The experimental and the simulated data are plotted with solid and dashed lines, respectively.

Table 2. The nearest atomic-pair distances deduced from the RMC-simulated structural models of $\mathrm{Zr}_{70} \mathrm{Pd}_{30} \mathrm{MG}$, the Goldschmidt atomic radii (SGAR) values, and the corresponding $\mathrm{Zr}_{2} \mathrm{Pd}_{1}$ crystalline alloy.

\begin{tabular}{cccc}
\hline \multirow{2}{*}{ Atomic Pairs } & \multicolumn{3}{c}{ Atomic-Pair Distances $(\AA) \mathbf{~} \mathbf{0 . 0 2}$} \\
\cline { 2 - 4 } & $\mathbf{Z r}_{\mathbf{7 0}} \mathbf{P d}_{\mathbf{3 0}} \mathbf{M G}$ & SGAR & Crystalline Alloy \\
\hline $\mathrm{Zr}-\mathrm{Zr}$ & 3.20 & 3.20 & 3.23 \\
$\mathrm{Zr}-\mathrm{Pd}$ & 2.90 & 2.97 & 3.06 \\
$\mathrm{Pd}-\mathrm{Pd}$ & 2.76 & 2.74 & 3.31 \\
$\mathrm{Pd}-\mathrm{Zr}$ & 2.90 & 2.97 & 3.06 \\
\hline
\end{tabular}

Furthermore, Voronoi tessellation [32] is carried out to analyze the RMC-simulated structural model, from which various indexed Voronoi clusters (VCs) can be obtained. Distributions of the major VCs centered with Zr or Pd atoms are plotted in Figure 3. In the present work, Zr atoms should be regarded as the solvents while Pd atoms should be the solutes, considering their concentrations. It is found that there are hundreds of types of VCs, and the most popular solvent-centered and solute-centered VCs are indexed as $\langle 0,2,8,4>,\langle 0,1,10,2\rangle,\langle 0,3,6,4\rangle$, and $\langle 0,2,8,2\rangle,<0,3,6,3\rangle$, $<0,2,8,1>$, respectively. This is consistent with the co-existence of various clusters presented in the theoretical work [5]. In each $<n 3, n 4, n 5, n 6>$ indexed VC, ni denotes the number $(n)$ of the $i$-fold 
rotation symmetry, and indicates the number of shell atoms connected with other $i$ shell atoms. In addition, the $\mathrm{CN}$ of the center atom in a $<n 3, n 4, n 5, n 6>\mathrm{VC}$ can be deduced because $\Sigma n i$ stands for the number of the shell atoms around the center. For instance, the center atom in a $<0,2,8,2>\mathrm{VC}$ has a CN of 12. As shown in Figure 3, the solvent and the solute have CNs ranging from 12-15 and 10-13, respectively. This indicates that the average $\mathrm{CN}$ around the solvent is obviously larger than its counterpart around the solute. According to the efficient cluster-packing model [33], the optimal CN relates to the size ratio between the center atom and the shell atoms in clusters. In our case, it is obvious that the $\mathrm{Zr}$ atom has a larger radius than that of the Pd atom. Therefore, it is reasonable that the Zr centers have more near neighbors.
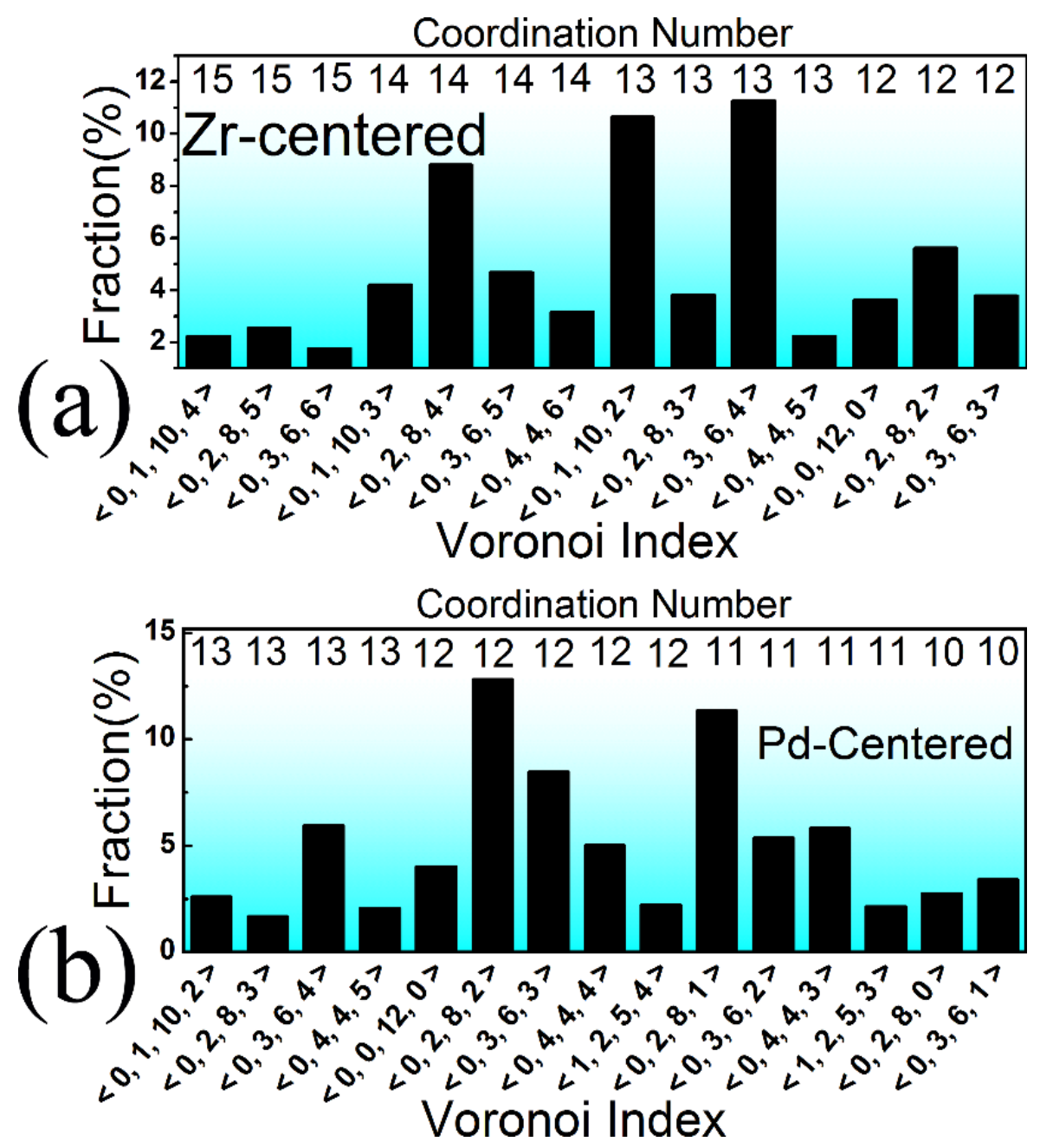

Figure 3. Distribution of the major Voronoi clusters centered with (a) $\mathrm{Zr}$ and (b) $\mathrm{Pd}$ atoms. Only those whose fractions are larger than $1.5 \%$ are selected. The $\mathrm{CN}$ value denotes the number of shell atoms of each VC, i.e., the CN around the center atom.

It has been pointed out that the ideal icosahedral or icosahedral-like VCs are indexed as $<0,0,12,0>$, $\langle 0,2,8,2\rangle,\langle 0,3,6,3\rangle,\langle 0,4,4,4>,<0,1,10,2\rangle$, and so on [32]. Configurations of these icosahedral or icosahedral-like VCs are plotted in Figure 4a-e. The icosahedron deduced from the $\mathrm{Zr}_{2} \mathrm{Pd}_{1} \mathrm{I}_{\text {-phase }}$ is also shown in Figure 4f, which is extremely similar with that shown in Figure 4a. It is observed in Figure 3 that icosahedral or icosahedral-like VCs have relatively high fractions. In particular, it is worth noting that the icosahedral-like VCs with a CN of 12 (such as $<0,2,8,2>$ and $<0,3,6,3>$ ) are some 
popular structural units centered with Pd atoms. According to the relationship between the $R$ value (the size ratio between the center and the shell atoms, where the optimal $R$ value is the so-called $R^{*}$ ) and the CN [29], the estimated $R$ value is close to the $R^{*}$ corresponding to a $\mathrm{CN}$ of 12 , which is in agreement with our result mentioned above.

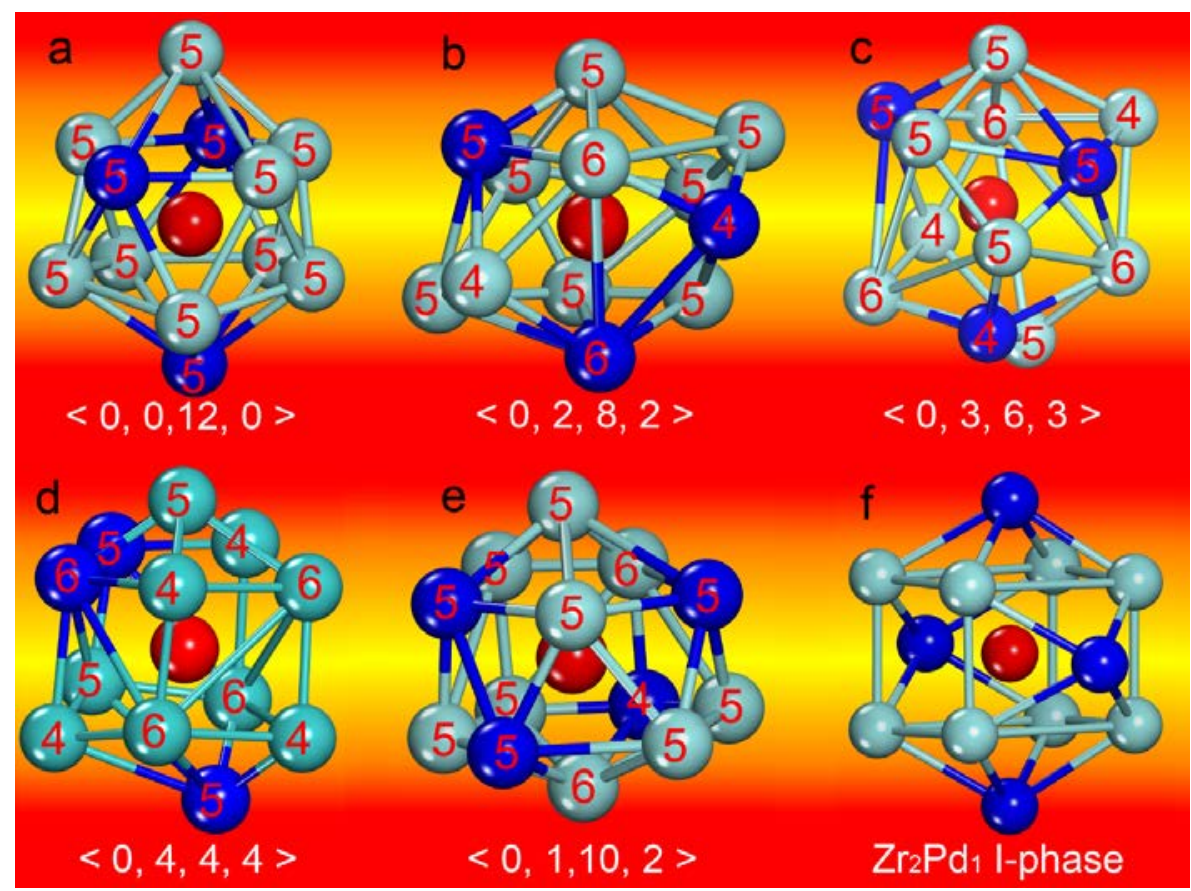

Figure 4. Configurations of the icosahedral or icosahedral-like VCs extracted from the RMC-simulated structural model, including: (a) $<0,0,12,0>$; (b) $<0,2,8,2>$; (c) $<0,3,6,3>$; (d) $<0,4,4,4>$; and (e) $<0,1,10,2>$. The number labeled on each shell atom stands for the number of its neighbor (connected) shell atoms, and also indicates the $i$-fold rotation symmetry. The icosahedron deduced from the $\mathrm{Zr}_{2} \mathrm{Pd}_{1} \mathrm{I}$-phase is shown in (f), which is extremely similar with that shown in (a).

Here we can explain why there is precipitation of an icosahedral quasicrystalline phase prior to phase transformation from glass to crystal when heating $\mathrm{Zr}_{70} \mathrm{Pd}_{30} \mathrm{MG}$. We have revealed that there are popular icosahedral or icosahedral-like VCs in the microstructure of $\mathrm{Zr}_{70} \mathrm{Pd}_{30} \mathrm{MG}$. There is not a large configuration discrepancy between VCs in the $\mathrm{Zr}_{70} \mathrm{Pd}_{30} \mathrm{MG}$ and those in the $\mathrm{Zr}_{2} \mathrm{Pd}_{1}$ I-phase, leading to their relatively small energy barrier of phase transformation. Therefore, the $\mathrm{Zr}_{2} \mathrm{Pd}_{1}$ I-phase is easy to form by rearranging and stacking those icosahedral or icosahedral-like VCs with quasi-periodicity during annealing. When adequate energy is provided to overcome the energy barrier during annealing, both the $\mathrm{Zr}_{2} \mathrm{Pd}_{1}$ I-phase and the residual amorphous phase will transform into the $\mathrm{Zr}_{2} \mathrm{Pd}_{1}$ tetragonal phase, as observed experimentally [19].

\section{Conclusions}

In summary, how synchrotron radiation techniques can be applied for detecting the microstructure in MG is studied. It is found that fitting the EXAFS signal of MG with a structural model directly cannot provide reliable structural information. A feasible scheme for investigating the microstructure 
of amorphous alloys is required. Combining synchrotron radiation-based experiments with simulations is tried in this work. It is revealed that the co-existence of various clusters is the intrinsic nature in the amorphous structure, and some icosahedral or icosahedral-like VCs are the popular structural units. This leads to their relatively small energy barrier for the amorphous-to-quasicrystal phase transformation, and can explain why there is precipitation of an icosahedral quasicrystalline phase prior to phase transformation from glass to crystal when annealing the $\mathrm{Zr}_{70} \mathrm{Pd}_{30} \mathrm{MG}$.

\section{Acknowledgments}

The authors would like to thank the HASYLAB in Germany, the Shanghai Synchrotron Radiation Facility in China, and the National Synchrotron Radiation Laboratory of China for the use of the advanced synchrotron radiation facilities. Financial support from the National Natural Science Foundation of China (Grant No. U1332112 and 51471088), the Fundamental Research Funds for the Central Universities (Grant No. NE2015004), the Funding for Outstanding Doctoral Dissertation in NUAA (Grant No. BCXJ12-08), the Funding of Jiangsu Innovation Program for Graduate Education (Grant No. CXLX13-152), and the project funded by the Priority Academic Program Development (PAPD) of Jiangsu Higher Education Institutions are gratefully acknowledged.

\section{Author Contributions}

G.-Q.G. performed simulation work upon the experimental data. L.Y. performed analysis of this work and wrote this article. S.-Y.W. and S.L. contributed to the experimental research work.

\section{Conflicts of Interest}

The authors declare no conflict of interest.

\section{References}

1. Cohen, M.H.; Turnbull, D. Metastability of amorphous structures. Nature 1964, 203, 964.

2. Finney, J.L. Modeling the structures of amorphous metals and alloys. Nature 1977, 266, 309-314.

3. Doye, J.P.K.; Wales, D.J. The structure and stability of atomic liquids: From clusters to bulk. Science 1996, 271, 484-487.

4. Tan, H.; Zhang, Y.; Ma, D.; Feng, Y.P.; Li, Y. Optimum glass formation at off-eutectic composition and its relation to skewed eutectic coupled zone in the La based $\mathrm{La}-\mathrm{Al}-(\mathrm{Cu}, \mathrm{Ni})$ pseudo ternary system. Acta Mater. 2003, 51, 4551-4561.

5. Sheng, H.W.; Luo, W.K.; Alamgir, F.M.; Bai, J.M.; Ma, E. Atomic packing and short-to-medium-range order in metallic glasses. Nature 2006, 439, 419-425.

6. Hirata, A.; Guan, P.F.; Fujita, T.; Hirotsu, Y.; Inoue, A.; Yavari, A.R.; Sakurai, T.; Chen, M.W. Direct observation of local atomic order in a metallic glass. Nat. Mater. 2011, 10, 28-33.

7. Wu, Z.W.; Li, M.Z.; Wang, W.H.; Liu, K.X. Hidden topological order and its correlation with glass-forming ability in metallic glasses. Nat. Commun. 2015, doi:10.1038/ncomms7035.

8. Bernal, J.D. Geometrical approach to the structure of liquids. Nature 1959, 183, 141-147. 
9. Gaskell, P.H. A new structural model for transition metal-metalloid glasses. Nature 1978, 276, 484-485.

10. Miracle, D.B. A structural model for metallic glasses. Nat. Mater. 2004, 3, 697-702.

11. Pfeiffer, F.; Weitkamp, T.; Bunk, O.; David, C. Phase retrieval and differential phase-contrast imaging with low-brilliance X-ray sources. Nat. Phys. 2006, 2, 258-261.

12. Lefebvre, S.; Quivy, A.; Bigot, J.; Calvayrac, Y.; Bellissent, R. A neutron diffraction determination of short-range order in a Ni63.7Zr36.3 glass. J. Phys. F 1985, 15, L99-L103.

13. Luo, W.K.; Sheng, H.W.; Alamgir, F.M.; Bai, J.M.; He, J.H.; Ma, E. Icosahedral Short-Range Order in Amorphous Alloys. Phys. Rev. Lett. 2004, doi:10.1103/PhysRevLett.92.145502.

14. Yang, L.; Xia, J.H.; Wang, Q.; Dong, C.; Chen, L.Y.; Ou, X.; Liu, J.F.; Jiang, J.Z.; Klementiev, K.; Saksl, K.; et al. Design of Cu8Zr5-based bulk metallic glasses. Appl. Phys. Lett. 2006, doi:10.1063/1.2213020.

15. Sadoc, J.F.; Dixmier, J. Structural investigation of amorphous CoP and NiP alloys by combined X-ray and neutron scattering. Mater. Sci. Eng. 1978, 23, 187-192.

16. Saksl, K.; Franz, H.; Jovari, P.; Klementiev, K.; Welter, E.; Ehnes, A.; Saida, J.; Inoue, A.; Jiang, J.Z. Evidence of icosahedral short-range order in $\mathrm{Zr}_{70} \mathrm{Cu}_{30}$ and $\mathrm{Zr}_{70} \mathrm{Cu}_{29} \mathrm{Pd}_{1}$ metallic glasses. Appl. Phys. Lett. 2003, 8333, 3924-3926.

17. Yang, L.; Guo, G.Q. Structural origin of the high glass-forming ability in Gd doped bulk metallic glasses. Appl. Phys. Lett. 2010, doi:10.1063/1.3485117.

18. Yang, L.; Guo, G.Q.; Chen, L.Y.; Wei, S.H.; Jiang, J.Z.; Wang, X.D. Atomic structure in Al-doped multicomponent bulk metallic glass. Scr. Mater. 2010, 63, 879-882.

19. Saida, J.; Kasai, M.; Matsubara, E.; Inoue, A. Stability of glassy state in Zr-based glassy alloys correlated with nano icosahedral phase formation. Ann. Chim. Sci. Mater. 2002, 27, 77-89.

20. Saida, J.; Matsushita, M.; Inoue, A. Direct observation of icosahedral cluster in $\mathrm{Zr}_{70} \mathrm{Pd}_{30}$ binary glassy alloy. Appl. Phys. Lett. 2002, 79, 412-414.

21. Xu, D.H.; Duan, G.; Johnson, W.L. Unusual glass-forming ability of bulk amorphous alloys based on ordinary metal copper. Phys. Rev. Lett. 2004, doi:10.1103/PhysRevLett.92.245504.

22. Takagi, T.; Ohkubo, T.; Hirotsu, Y.; Murty, B.S.; Hono, K.; Shindo, D. Local structure of amorphous $\mathrm{Zr}_{70} \mathrm{Pd}_{30}$ alloy studied by electron diffraction. Appl. Phys. Lett. 2001, 79, 485-487.

23. Liss, K.D.; Bartels, A.; Schreyer, A.; Clemens, H. High energy X-rays: A tool for advanced bulk investigations in materials science and physics. Textures Microstruct. 2003, 35, 219-252.

24. Hammersley, A.P.; Svensson, S.O.; Hanfland, M.; Fitch, A.N.; Häusermann, D. Two-dimensional detector software: From real detector to idealised image or two-theta scan. High Press. Res. 1996, 14, 235-248.

25. Faber, T.E.; Ziman, J.M. A theory of the electrical properties of liquid metals. Philos. Mag. 1965, 11, 153-173.

26. Klementev, K.V. Extraction of the fine structure from X-ray absorption spectra. J. Phys. D 2001, 34, 209-217.

27. Yang, L.; Guo, G.Q.; Chen, L.Y.; Huang, C.L.; Ge, T.; Chen, D.; Liaw, P.K.; Saksl, K.; Ren, Y.; Zeng, Q.S.; et al. Atomic-Scale Mechanisms of the Glass-Forming Ability in Metallic Glasses. Phys. Rev. Lett. 2012, doi:10.1103/PhysRevLett.109.105502. 
28. McGreevy, R.L.; Pusztai, L. Reverse Monte Carlo Simulation: A new technique for the determination of disordered structures. Mol. Simul. 1988, 1, 359-367.

29. Yang, L.; Guo, G.Q.; Jiang, J.Z.; Chen, L.Y.; Wei, S.H. "Soft" atoms in $\mathrm{Zr}_{70} \mathrm{Pd}_{30}$ metal-metal amorphous alloy. Scr. Mater. 2010, 63, 883-886.

30. Takahara, Y.; Narita, N. Local electronic structures and chemical bonds in Zr-based metallic glasses. Mater. Trans. 2004, 45, 1172-1176.

31. Huang, L.; Wang, C.Z.; Hao, S.G.; Kramer, M.J.; Ho, K.M. Atomic size and chemical effects on the local order of $\mathrm{Zr}_{2} \mathrm{M}(\mathrm{M}=\mathrm{Co}, \mathrm{Ni}, \mathrm{Cu}$, and $\mathrm{Ag}$ ) binary liquids. Phys. Rev. B 2010, doi:10.1103/PhysRevB.81.014108.

32. Wang, S.Y.; Kramer, M.J.; Xu, M.; Wu, S.; Hao, S.G.; Sordelet, D.J.; Ho, K.M.; Wang, C.Z. Experimental and $a b$ initio molecular dynamics simulation studies of liquid $\mathrm{Al}_{60} \mathrm{Cu}_{40}$ alloy. Phys. Rev. B 2009, doi:10.1103/PhysRevB.79.144205.

33. Miracle, D.B.; Sanders, W.S.; Senkov, O.N. The influence of efficient atomic packing on the constitution of metallic glasses. Philos. Mag. 2003, 83, 2409-2428.

(C) 2015 by the authors; licensee MDPI, Basel, Switzerland. This article is an open access article distributed under the terms and conditions of the Creative Commons Attribution license (http://creativecommons.org/licenses/by/4.0/). 